\title{
Retraction Note to: The Semaphorin 4D-Plexin-B1-RhoA signaling axis recruits pericytes and regulates vascular permeability through endothelial production of PDGF-B and ANGPTL4
}

\author{
Hua Zhou ${ }^{1} \cdot$ Ying-Hua Yang ${ }^{1} \cdot$ John R. Basile ${ }^{1,2}$
}

Published online: 26 February 2020

C Springer Nature B.V. 2020

\section{Retraction to: Angiogenesis (2014) 17:261-274 https://doi.org/10.1007/s10456-013-9395-0}

The Editors-in-Chief have retracted this article [1] following an investigation by the University of Maryland. The institution found that in Fig. 1c, the graph showing PDGF-B does not match the original data for the 24 -h time point. The graph shows the value to be over $1000 \mathrm{pg} / \mathrm{ml}$, but the original data have a value of 106.626. In Fig. 1f, the data were entered manually to create the standard deviation bars. The data manually entered do not match the original data. When the standard deviations for the original data were calculated, the $p$ values were no longer significant using a paired Student $t$ test. In Fig. 2c, the original data do not match the published data. In Fig. 4b, the images in the first lane and the fifth lane are from the same micrograph (i.e., the same set of conditions). However, the published figure claims that they are different conditions. The metadata in this figure also shows different cell lines than those noted in the article. The first and last images are labelled as "Du145 shAR3 anti
AR3.jpg". The second image is labelled as "Du145 shAR8 anti AR8.jpg". The third image is labelled as "Cos1 mARs3 mS3-2 antibody-2.jpg." The fourth image is labelled as "R1 3634 bleed.jpg". Due to these errors, the Editors-in-Chief have found that the results are no longer reliable.

John R. Basile agrees to this retraction. Hua Zhou and Ying-Hua Yang have not responded to any correspondence from the publisher about this retraction.

\section{Reference}

1. Zhou H, Yang YH, Basile JR (2014) The Semaphorin 4D-Plexin$\mathrm{B} 1-\mathrm{RhoA}$ signaling axis recruits pericytes and regulates vascular permeability through endothelial production of PDGF-B and ANGPTL4. Angiogenesis 17:261-274. https://doi.org/10.1007/ s10456-013-9395-0

Publisher's Note Springer Nature remains neutral with regard to jurisdictional claims in published maps and institutional affiliations.
The original article can be found online at https://doi.org/10.1007/ s10456-013-9395-0.

John R. Basile

jbasile@umaryland.edu

1 Department of Oncology and Diagnostic Sciences, University of Maryland Dental School, 650 West Baltimore Street, 7-North, Baltimore, MD 21201, USA

2 Greenebaum Cancer Center, 22 S. Greene Street, Baltimore, MD 21201, USA 